\title{
Reducing nosocomial transmission of COVID-19: implementation of a COVID-19 triage system
}

\author{
Authors: Rachel M Wake, ${ }^{A}$ Matthew Morgan, ${ }^{B}$ Jenny Choi ${ }^{B}$ and Simon Winn ${ }^{C}$
}

\begin{abstract}
Nosocomial transmission of COVID-19 puts patients with other medical problems at risk of severe illness and death. Of 662 inpatients with COVID-19 at an NHS Trust in South London, 45 (6.8\%) were likely to have acquired COVID-19 in hospital. These patients had no evidence of respiratory or influenza-like illness on admission and developed symptoms, with positive SARS-CoV-2 PCR test results, more than 7 days after admission ( $>14$ days for 38 [5.7\%] patients). Forty ( $88.9 \%$ ) of these patients had shared a ward with a confirmed COVID-19 case prior to testing positive. Implementation of a triage system combining clinical assessment with rapid SARS-CoV-2 testing facilitated cohorting so that fewer susceptible patients were exposed to COVID-19 on shared wards. With hospital service resumption alongside the possibility of future waves of COVID-19 related admissions, strategies to prevent nosocomial transmission are essential. Point-of-care diagnostics can complement clinical assessment to rapidly identify patients with COVID-19 and reduce risk of transmission within hospitals.
\end{abstract}

KEYWORDS: COVID-19, nosocomial infection, infection control, point-of-care testing, patient isolation

DOI: 10.7861/clinmed.2020-0411

\section{Background and aims}

Patients with existing medical conditions are at greatest risk of severe illness and death following infection with SARS-CoV-2., ${ }^{1,2}$ Rapid identification of patients with COVID-19 and prevention of nosocomial transmission is therefore of critical importance. Universal screening using SARS-CoV-2 real-time reverse transcription-polymerase chain reaction assays ( $r R T P C R$ ) is now advised for every patient admitted to hospital in the UK. However, with delayed result availability and the relatively poor sensitivity of SARS-CoV-2 PCR tests, ${ }^{3,4}$ testing alone is not an adequate screening tool to prevent transmission; effective clinical triage and cohorting is required.

Authors: Aacademic clinical lecturer, St George's, University of London, London, UK; Bfoundation doctor, Epsom and St Helier University Hospitals NHS Trust, Epsom, UK; ' Cacute medicine divisional medical director, Epsom and St Helier University Hospitals NHS Trust, Epsom, UK
During March and April 2020, incidence of patients with COVID-19 exceeded availability of single isolation rooms in many UK hospitals; cohorting of patients with suspected or confirmed COVID-19 was advised. ${ }^{5}$ Clinical cohorting resulted in patients presenting with unrelated respiratory or influenza-like illnesses being admitted to 'COVID' wards and patients with undiagnosed asymptomatic, presymptomatic or atypical COVID-19 being admitted to "nonCOVID' wards alongside patients with unrelated medical problems. Susceptible individuals were therefore exposed to SARS-CoV-2 by close proximity with patients transmitting virus in droplet or aerosol form, by environmental and fomite contamination of shared facilities, or by infected healthcare workers.

To understand the risk of nosocomial transmission at an NHS hospital trust in South London, we assessed clinical features at admission, and time between admission and testing for patients diagnosed with COVID-19 between 11 March 2020 and 12 May 2020. Data were collected as part of the ISARIC Clinical Characterisation Protocol approved by the NHS Health Research Authority.

A COVID-19 triage system was subsequently implemented and is outlined here, combining a clinical assessment tool with targeted rapid SARS-CoV-2 PCR testing to reduce the risk of transmission to susceptible patients in hospital.

Assessing the prevalence of nosocomial transmission in our Trust

During the test period, 662 patients tested positive for SARS-CoV-2 infection during their admission to hospital. A majority (573, 86.5\%) were admitted with clinical features of COVID-19, and tested positive within the first 7 days of admission (community-acquired COVID-19). The remaining 89 (13.4\%) patients were tested positive for COVID-19 following more than 7 days in hospital and therefore fit the criteria for probable (positive on day $8-14 ; 34$ [5.1\%]) or definite (positive after day 14; 55 [8.3\%]) hospital-associated COVID-19, using NHS surveillance criteria of hospital-acquired infections in England. ${ }^{6}$

On analysis of documented clinical features, we found that $44(49.4 \%)$ of the 89 patients defined as 'hospital-associated' by NHS surveillance criteria may have in fact acquired COVID-19 in the community: 28 were admitted with evidence of a respiratory or influenza-like illness or radiological changes consistent with pneumonia, but were not tested for COVID-19 until after 7 days of admission; 16 developed symptoms and were tested for COVID-19 within the first 7 days of admission, but the result of their initial test was negative. 
662 patients with COVID-19 during hopsitalisation

573 communityacquired COVID-19

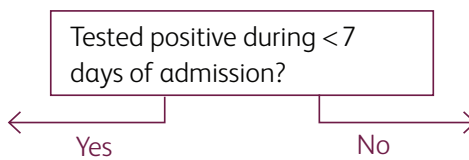

44 possible communityacquired COVID-19

\section{9 hospital-associated}

COVID-19

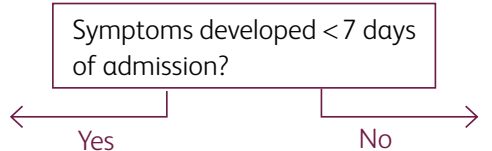

8 likely hospitalacquired

\section{5 hospital-acquired COVID-19}

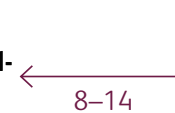

Days of hospitalisation before symptom onset?

Fig 1. Case categorisation. Flow chart to show categorisation of cases as hospital-associated COVID-19 if the patient had a positive test following $>7$ days in hospital, and likely or highly likely hospital-acquired COVID-19 if they had a positive test and symptom onset following $>7$ days in hospital.

Using the more conservative criteria of date of symptom onset rather than date of positive test, we estimate that 45 patients (6.7\% of cases) acquired COVID-19 in hospital (see Fig 1). These patients were admitted to hospital for unrelated medical problems including fall-related injuries (12), and neurological (12), gastrointestinal (8), cardiac (3) or other (10) medical problems. Clinical features of COVID-19, with subsequent positive test results for SARS-CoV-2, developed during day $8-14$ in 8 patients $(1.2 \%$, likely hospital-acquired) and following more than 14 days in hospital in 39 patients (5.8\%, highly likely hospital-acquired). Of the 45 patients with hospital-acquired COVID-19, median age was 81 (IQR 71-87), 22 (48.9\%) were male, 39 (86.7\%) had underlying comorbidities, and 5/42 (11.9\%) were recorded as Black, Asian or other minority ethnicity. Median length of stay was 33 days (IQR
22-55), during which two (4.4\%) patients were admitted to ITU, and $14(31.1 \%)$ patients died. Death was not more likely in this group than patients who tested COVID-19 positive within 7 days of admission (likely community-acquired) during the same period (208/573, 36.3\%, $p=0.5)$.

Hospital transmission of COVID-19 is widely recognised ${ }^{7-10}$ and although mode of infection remains unclear, ward-based transmission is highly suspected. Genomic surveillance of hospitalacquired SARS-CoV-2 among patients has confirmed nosocomial transmission between patients sharing wards. ${ }^{8}$ Although analysis for genetic linkage was not possible in this cohort, 40 (88.9\%) of patients with hospital-acquired infection shared a ward with a confirmed COVID-19 positive patient within the previous 14 days, and $13(28.9 \%$ ) were sharing a bay (Fig 2). The remaining

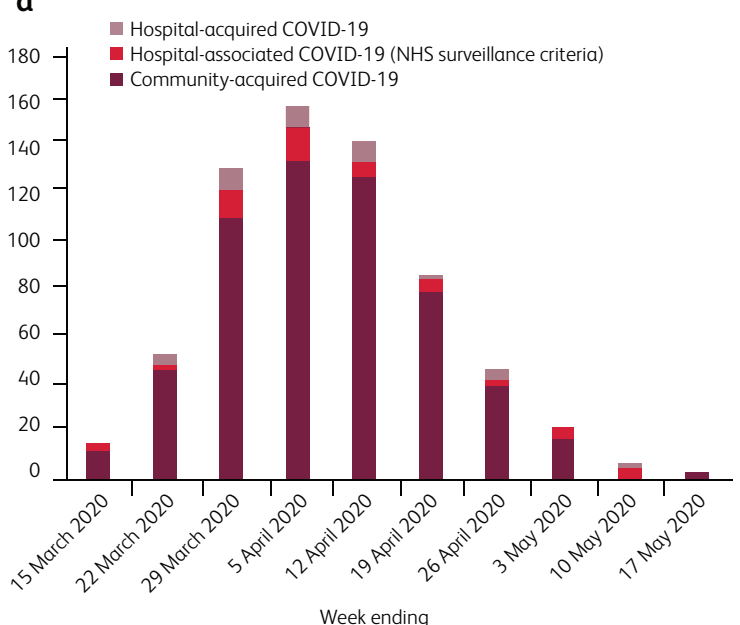

b

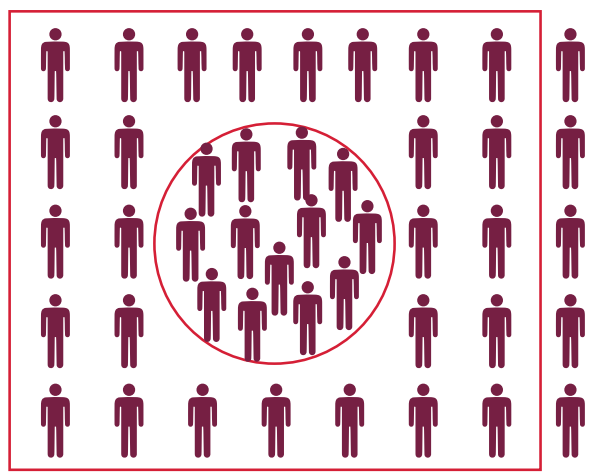

Fig 2. Proportion of cases categorised as hospital-associated and hospital-acquired and patterns of ward and bay sharing prior to infection. a) Bar chart to show the total number of patients admitted to hospital with COVID-19 by week, the proportion of cases that were categorised as 'hospital-associated' COVID-19 using NHS surveillance criteria of time from admission to positive test ( $>7$ days), and the proportion that were categorised as 'hospital-acquired' COVID-19 using time from admission to symptom onset ( $>7$ days). b) Diagram to show the number of patients with hospital-acquired COVID-19 who during the 14 days prior to testing positive were located in wards (box) or bays (circle) with confirmed COVID-19 patients who were likely to be infectious (during the 14 days after they tested positive). 40 (88.9\%) patients were located on wards with COVID-19 cases during the 14 days prior to testing positive; 13 (28.9\%) were located in the same bays. 

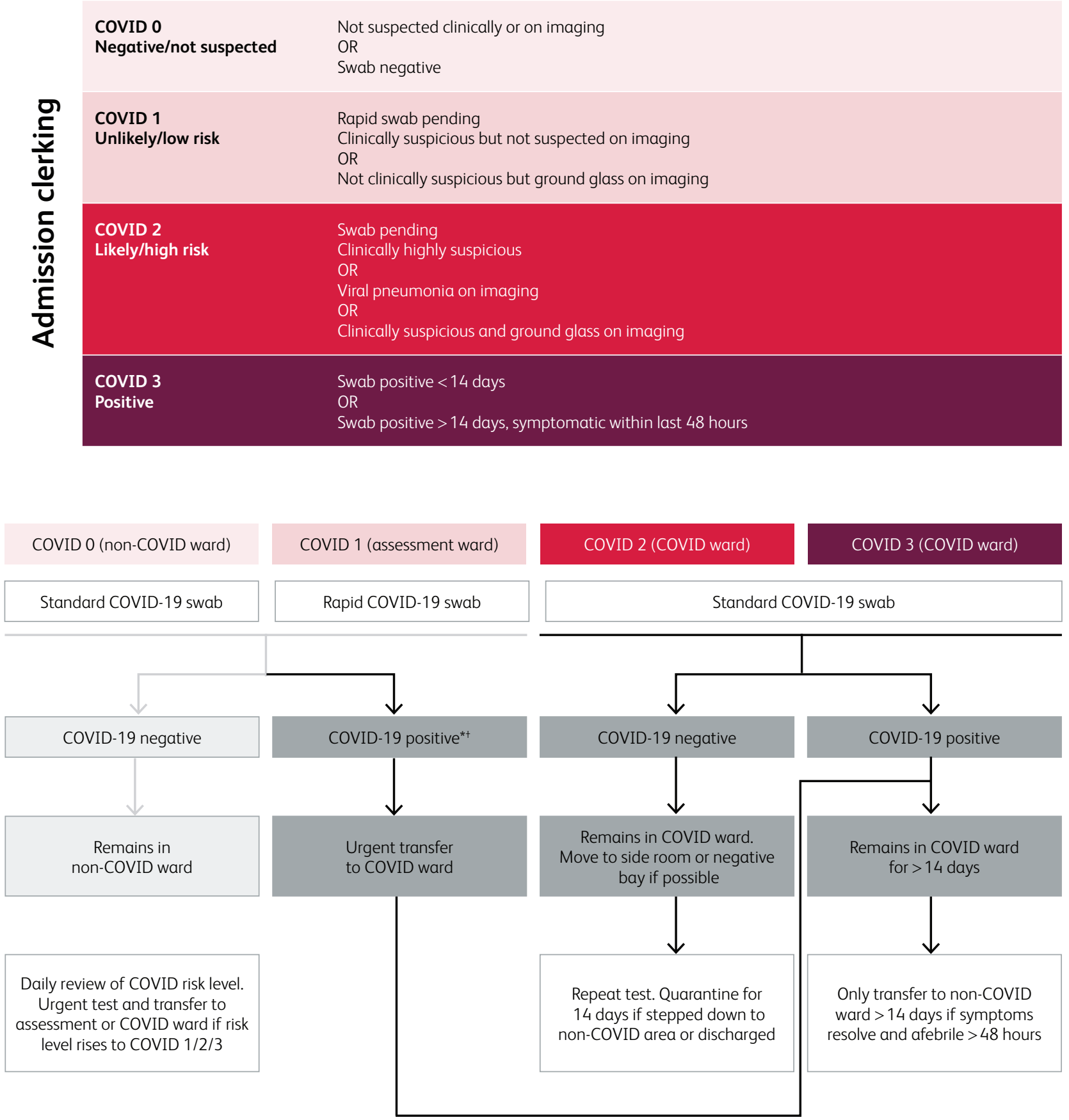

* Patients who receive a positive COVID test result but have a low clinical sispicion of COVID-19 may present a false positive and should be transferred to a side room on a COVID ward if possible

+ Contacts of patients testing COVID-19 positive in non-COVID wards (eg sharing bays) should be isolated or cohorted in a closed bay for 14 days and monitored closely for new clinical features of COVID-19

Fig 3. COVID-19 Likelihood Scoring Tool and flow-chart to guide testing and cohorting of patients admitted to hospital. 
$5(11.1 \%)$ patients may have acquired COVID-19 from other shared hospital facilities or from infected health care workers.

\section{A clinical assessment tool with rapid targeted testing for COVID-19 triage and cohorting}

To prevent nosocomial transmission, we implemented a clinical assessment tool to improve the effectiveness of COVID-19 triage and cohorting (Fig 3). Since routine SARS-CoV-2 PCR testing has relatively low sensitivity ${ }^{3,11,12}$ and turnaround times can be prolonged, this tool relies on clinical history and findings, laboratory results and radiology to categorise patients according to clinical likelihood of COVID-19. Patients are then triaged to separate wards; patients with no symptoms or signs of COVID-19 (likelihood score 0 ) are separated from those with low grade suspicion of COVID-19 (score 1) and those with high grade suspicion (score 2) or confirmed COVID-19 (score 3) are cohorted together on a separate ward. Patients with low-grade suspicion (score 1) are cared for in isolation rooms when possible and prioritised for rapid SARS-CoV-2 PCR testing to establish their transfer destination. In this way, positive patients are separated from negative patients as early as possible. Isolation rooms are also prioritised for those with an incongruous clinical presentation (possible test inaccuracy) and those with significant risk factors for severe illness such as immunosuppression. Patients are then re-assessed daily to ensure that anyone incubating infection is identified and transferred to an appropriate ward, minimising exposure to other patients.

We performed a point prevalence survey of clinicians caring for patients on four COVID-19 cohort wards before and after implementation of the clinical assessment tool. Prior to implementation there were 38 confirmed or highly suspected COVID-19 cases and 30 patients with no or low-grade suspicion of COVID-19 being cared for on these wards. All 30 (100\%) patients with unrelated illnesses were sharing wards with confirmed COVID-19 patients; 13 (43\%) within open bays. Two weeks after implementation of the assessment tool, two of the four wards had been re-designated for patients with a low-grade suspicion of COVID-19. There were 16 confirmed or highly suspected COVID-19 patients and 55 with no or low-grade suspicion of COVID-19. $12(22 \%)$ patients with no or low-grade suspicion of COVID-19 at the time of the survey were sharing wards with confirmed COVID-19 patients; four (7\%) within open bays. These patients were subsequently moved to side-rooms when available to reduce risk of exposure.

Alongside clinical triage, rapid screening tests are required to prevent nosocomial transmission. Universal screening of all hospital admissions identifies asymptomatic and pre-symptomatic patients with potential to transmit infection. ${ }^{9,13-16}$ However, in our Trust, median turnaround time between taking samples and obtaining results was 2 days (range $0-11$ days) during March and April 2020. Rapid PCR tests for COVID-19, such as the SAMBA II (DRW, Cambridge, UK) and the Xpert Xpress (Cepheid, CA, USA) have the potential to identify patients with COVID-19 within 30-90 minutes at the point of care, ${ }^{17}$ facilitating early isolation and minimising exposure to susceptible patients. Within our Trust, patients with a low clinical likelihood of COVID-19 are prioritised for testing using SAMBA II (DRW, Cambridge, UK). This complements the triage tool by facilitating urgent transfer of patients to appropriate clinical areas within hours of arrival.

Implementation of clinical triage and targeted rapid testing reduced mixing of confirmed and suspected COVID-19 cases with susceptible patients, although a fifth of patients with no or low clinical suspicion remained on COVID-19 cohort wards. This is likely due to limited availability of isolation rooms for patients who had come in contact with COVID-19 and were potentially incubating infection. These patients may have been assessed as likely COVID-19 on admission but re-categorised prior to the survey following additional clinical information indicating alternative diagnoses.

As well as ward-based contact with COVID-19 cases, healthcare workers are another potential source of hospital-acquired infection. ${ }^{8,9,16}$ Screening at London hospitals found that the SARSCoV-2 prevalence peaked at $7.1 \%$ in asymptomatic health-care workers ${ }^{18}$ and that over $45 \%$ became seropositive for SARS-CoV-2 antibodies during March and April 2020.19 Since pre-and asymptomatic transmission is possible, ${ }^{9,14}$ universal face-mask use is likely to reduce transmission. Additionally, regular SARS-CoV-2 screening among all patient-facing staff warrants consideration.

\section{Conclusion}

While social distancing measures have reduced transmission of COVID-19 in the community, nosocomial transmission continues to put vulnerable populations at risk of severe illness and death. We found that around 1 in 15 patients acquired COVID-19 in hospital during the study period, using both clinical and timeto-diagnosis criteria. This was around half of those fitting NHS surveillance criteria alone. As hospital services return to a new normal, prevention of nosocomial transmission remains vitally important, particularly since this may coincide with a new wave of COVID-19 related admissions. We demonstrate how a simple clinical assessment tool together with targeted use of rapid PCR testing can optimise use of the bed base for patients with and without COVID-19. While incidence of COVID-19 remains low, clinical teams and bed management operations must develop locally appropriate systems to protect susceptible patients during admission to hospital, using clinical expertise and point of care testing to rapidly identify and cohort patients with COVID-19.

\section{References}

1 Zhou F, Yu T, Du R et al. Clinical course and risk factors for mortality of adult inpatients with COVID-19 in Wuhan, China: a retrospective cohort study. Lancet 395:1054-62.

2 Wu C, Chen X, Cai Y et al. Risk factors associated with acute respiratory distress syndrome and death in patients with coronavirus disease 2019 pneumonia in Wuhan, China. JAMA Intern Med 2020;180:1-11.

3 Winichakoon P, Chaiwarith R, Liwsrisakun C et al. Negative nasopharyngeal and oropharyngeal swab does not rule out COVID-19. J Clin Microbiol 2020;58:e00297-20.

4 Han H, Luo Q, Mo F, Long L, Zheng W. SARS-CoV-2 RNA more readily detected in induced sputum than in throat swabs of convalescent COVID-19 patients. Lancet Infect Dis 2020;20:655-6.

5 Public Health England. COVID-19 infection prevention and control guidance. PHE, 2020. https://assets.publishing.service.gov.uk/ government/uploads/system/uploads/attachment_data/file/ 886668/COVID-19_Infection_prevention_and_control_guidance_ complete.pdf [Accessed 21 May 2020].

6 May R. Interim data collection - hospital-onset COVID-19. Publication Approval Reference 001559. NHS England and NHS Improvement, 2020.

7 McMichael TM, Currie DW et al. Epidemiology of Covid-19 in a long-term care facility in King County, Washington. N Engl ] Med 2020;382:2005-11. 
8 Meredith LW, Hamilton WL, Warne B et al. Rapid implementation of real-time SARS-CoV-2 sequencing to investigate healthcare-associated COVID-19 Infections. medRxiv 2020, 2020.05.08.20095687.

9 Arons MM, Hatfield KM, Reddy SC et al. Presymptomatic SARS-CoV-2 infections and transmission in a skilled nursing facility. N Engl J Med 2020;382:2081-90.

10 Rickman HM, Rampling T, Shaw K et al. Nosocomial transmission of COVID-19: a retrospective study of 66 hospital-acquired cases in a London teaching hospital. Clin Infect Dis 2020, in press (doi: 10.1093/cid/ciaa816).

11 Zou L, Ruan F, Huang M et al. SARS-CoV-2 viral load in upper respiratory specimens of infected patients. N Engl ] Med 2020; 382:1177-9.

12 Ye G, Li Y, Lu M et al. Experience of different upper respiratory tract sampling strategies for detection of COVID-19. J Hosp Infect 2020;105:1-2.

13 Nishiura H, Kobayashi T, Suzuki A et al. Estimation of the asymptomatic ratio of novel coronavirus infections (COVID-19). Int J Infect Dis 2020;94:154-5.

14 Qian G, Yang N, Ma AHY et al. A COVID-19 transmission within a family cluster by presymptomatic infectors in China. Clin Infect Dis 2020, in press (doi: 10.1093/cid/ciaa316).
15 Wei WE, Li Z, Chiew C] et al. Presymptomatic transmission of SARS-CoV-2 - Singapore, January 23-March 16, 2020. MMWR Morb Mortal Wkly Rep 2020;69:411-5.

16 Rivett L, Sridhar S, Sparkes D et al. Screening of healthcare workers for SARS-CoV-2 highlights the role of asymptomatic carriage in COVID-19 transmission. elife 2020;9:e58728.

17 Collier DA, Assennato SM, Sithole $\mathrm{N}$ et al. Rapid point of care nucleic acid testing for SARS-CoV-2 in hospitalised patients: a clinical trial and implementation study. medRxiv 2020, 2020.05.31.20114520.

18 Treibel TA, Manisty C, Burton M et al. COVID-19: PCR screening of asymptomatic health-care workers at London hospital. Lancet 2020;395:1608-10.

19 Houlihan C, Vora N, Byrne T et al. SARS-CoV-2 virus and antibodies in front-line health care workers in an acute hospital in London: preliminary results from a longitudinal study. medRxiv 2020, 2020.06.08.20120584.

Address for correspondence: Dr Rachel M Wake, Institute for Infection \& Immunity, St George's, University of London, Cranmer Terrace, London SW17 ORE, UK.

Email: rachel.wake1@nhs.net 